\title{
Narrative evaluation in patient feedback
}

\section{A study of online comments about UK healthcare services}

\author{
Gavin Brookes, Tony McEnery,, Mark McGlashan, \\ Gillian Smith ${ }^{1}$ and Mark Wilkinson ${ }^{1}$ \\ ${ }^{1}$ Lancaster University $\mid{ }^{2}$ Xian Jiaotong University $\mid{ }^{3}$ Birmingham City \\ University
}

This study examines how patients use narratives to evaluate their experiences of healthcare services online. The analysis draws on corpus linguistic techniques, specifically annotation, applying Labov and Waletzky's (1967) framework to a sample of online comments about the NHS in England. Narratives are pervasive in this context, being present more than absent in the patients' comments, but are particularly prominent in comments which evaluate care negatively. Evaluations can be accomplished through all the structural elements of the narrative, including in combination with one another. However, the presence and ordering of these elements does not seem to be influenced by the type of evaluation given (i.e. positive, negative or more neutral). As mediated social practice, the narratives are shaped by the technological affordances and social dynamics of this context, for instance in the placement of particular structural elements and the design of narratives for particular "imagined" audiences.

Keywords: patient feedback, Health Communication, NHS, narrative, annotation

\section{Introduction}

While a substantial body of research has examined narratives with a view to understanding individuals' experiences of health and illness, in this article we analyse narratives in patients' feedback about healthcare services - a context which has yet to be explored through a narrative lens. We explore patient feedback about the National Health Service in England in the form of online comments submitted to the NHS Choices website. The starting point for this study is the 
view of narrative as a form of social practice which "captures habituality and regularity in discourse in the sense of recurrent evolving responses to given situations while allowing for emergence and situational contingency" (Georgakopoulou, 2007: 18). Hence, we view narratives not simply as discursive accounts of past experiences and events but as situated activities capable of fulfilling a range of social functions.

Studies of narrative social practices in organisational contexts have highlighted their potential to perform a range of complex social functions, including to construct individual and group identity, establish shared values, and educate, instruct, and entertain (e.g. Holmes \& Marra, 2005). While our analysis is receptive to the range of social functions that can be accomplished using narratives within this context, we are particularly interested in how narrative social practices contribute to another social practice, that of evaluation, following Linde (1997: 166) who views evaluation as a form of social practice which "constitutes the social determination of the meanings of one's self, one's actions, and one's environment”.

To study the intersection of narrative and evaluative social practices in online patient feedback texts, we draw on corpus linguistics - a collection of methods used to cycle between quantitative and qualitative linguistic analyses of large collections of naturally occurring language data (Brookes \& McEnery, 2020) and specifically the use of annotation (introduced later). Our analysis seeks to answer the following questions: (i.) How prevalent is narrative in online patient feedback? (ii.) Which structural narrative elements are used to perform evaluative social practices? and (iii.) Do narrative structures alter depending on the evaluations they perform (i.e. as positive or negative)? This article therefore sheds new light on narratives as social practices in an organisational context in which they have presently received limited attention (in online patient feedback) and provides new insights, which are likely to apply to other organisational contexts, regarding the capacity for narratives to perform the social practice of evaluation.

\section{Background}

Narratives represent a rich resource in illness and healthcare contexts with respect to practice and research. For health professionals, narratives can provide means through which they can acquire and perform knowledge and establish their institutional roles and identities (Sarangi \& Clarke, 2002). For patients, narratives can be used to share experiences of health and illness. Engaging with patients' narratives can thus help health practitioners to gain greater a understanding of patients' health-related knowledge and experiences. In turn they can contribute 
to improving the accuracy and efficiency of diagnosis and treatment, informing the education of patients and professionals, and helping professionals to set a patient-centred agenda and strengthen their therapeutic alliances with patients (Greenhalgh \& Hurwitz, 1999). For researchers, narratives, particularly when viewed as forms of social practice, afford the opportunity to study how individuals make sense of their experiences of illness and craft and enact identities (Harvey \& Koteyko, 2012).

Within the healthcare domain, "narrative competence" (Charon, 2006) has emerged as a clinical skill for healthcare professionals in medicine, nursing, social work and psychotherapy (Arribas-Ayllon, 2021). The "narrative based medicine" movement (Greenhalgh, 1999) recognizes narratives as valuable to individuals (patients) coming to terms with their illnesses. It can also improve the accuracy of diagnoses and appropriateness of treatments, and benefit the therapeutic alliance between patients and professionals (Greenhalgh \& Hurwitz, 1999). More generally, health-related research in the sciences, social sciences, arts and humanities has witnessed a "narrative turn" (Polkinghorne, 1988) which has given rise to a diverse range of theoretical and methodological perspectives on narrative. These employ distinct underlying ontologies and thus conceptions of what narratives are, what functions they can and do perform, and what an analysis of them can show. The earliest work focused on narratives told by people affected by particular illnesses (particularly chronic illnesses) and other health-related issues, with the narratives studied as a means of learning about, inter alia, how individuals make sense of their experiences of illness (Kleinman, 1988), how illness narratives fit within grander cultural narratives (Frank, 1995), the co-constructedness of narratives between patients and doctors (Clark \& Mishler, 1992), and how narratives contribute to the construction (and reconstruction) of social identities in relation to ill-health (Riessman, 1990).

However, many studies have been criticised for not always taking a systematic approach to the study of narrative. For example, Atkinson and Delamont (2006) describe the tendency for studies adopting narrative-based approaches to regard their data uncritically, treating the stories told by their informants, unproblematically, as a transparent window on the teller's experiences, their state of mind and even their "authentic" self. Such approaches are problematic, as not only do they assume the existence of a single, "authentic" self (that can be reliably accessed through language), but they also overlook the fact that language use can - and usually is - shaped by motivations other than simply being "transparent" or "authentic"; language can be used to deceive or manipulate, for example. Language can be used to project certain versions of ourselves or the events we are recounting that we want to be seen by others, and these can be consistent but also knowingly incongruous with the events being described and our roles or attitudes 
in relation to them. Another problematic trend is the tendency for narratives to be treated as "freefloating, decontextualized entities rather than as socially occasioned instances of discourse" (Jones, 2013:116). Indeed, most studies of illness narratives are based on language produced in the context of research interviews, as opposed to contexts in which discourse is produced naturally. However, the possible effects of this research context on the narratives being relayed is rarely if ever addressed.

Narratives must therefore be analysed "in terms of their rhetorical, persuasive properties, and their functions in constructing particular versions of events, justifications of actions, evaluations of others, and so on" (Atkinson and Delamont, 2006:167). Analysts should also be mindful of the influence of the particular socio-cultural and textual contexts in which narratives are produced and by which they are constituted (and of which they are constative). This is where the view of narratives as social practice can, and has, contributed usefully to the study of narratives in illness and health(care) contexts. For example, in their study of a genetic counselling session, Sarangi and Clarke (2002) describe how narratives are jointly constructed in a way that is designed to legitimate actions in decisionmaking. Harvey and Koteyko (2012:88) argue that modern societies are characterised by "complex institutional cultures that display a wide variety of narrative forms, whether written, spoken or digital", while observing that online platforms have "led to a creation of new conventions and norms of narrative and narration" (ibid.). Indeed, online, computer-mediated contexts have provided rich sources for applied linguists and discourse analysts interested in examining narrative practices in relation to illness and health(care). Studies in this vein (e.g. Lamerichs \& Te Molder, 2003) have demonstrated narrative practices relating to illness and health(care) to be deeply entwined with users' interpersonal and interactional concerns, with narratives constructed in ways which, for example, help to establish credibility, engender responses from others, and protect their "face" (Goffman, 1955) by attenuating those aspects of their selves or their illness experiences that they perceive to be likely to evoke critical, even stigmatising responses from others.

While the study of health(care) and illness narratives has been enriched by more theoretically informed engagement with the socio-cultural and textual contexts in which narrative practices are situated, challenges remain. For example, narratives gathered, particularly in purely qualitative studies, are not necessarily representative of the wider population or context being studied and thus provide limited insight into the distribution or frequency of observed features or characteristics (Ziebland, 2013:45). The challenge of increasing the scope and generalizability of narrative studies is also one of retaining scope for including a theory-sensitive account of narrative practices. We approach this challenge by 
analysing patients' narratives of their experiences of healthcare services utilising a corpus-based approach to narrative analysis. This approach brings together quantitative with qualitative perspectives on the data to add scalability to the analysis of the narratives within it, while retaining a commitment to narrative theory. Before outlining our data and analytical approach in the next section, we first introduce the concepts of patient feedback and evaluative social practices. As part of this discussion, consistent with our view of both narrative and evaluation as contextually situated social practices, we also lay the groundwork for our analysis by describing the context in which the specific feedback in our data was given.

In England, since 2002 all NHS trusts have been required to gather feedback about their services from patients and report annually on the results of this feedback to the regulatory body, the Healthcare Commission. The present study focuses on feedback submitted in the form of comments posted on the NHS Choices website.

Patient feedback is a characteristically evaluative genre, since the purpose of texts like the comments posted to the NHS Choices website is for patients to evaluate the services that they have used. We follow Linde (1997) and view evaluation as a form of social practice, and one that has the potential to be carried out by narrative. At this point it is important to acknowledge the distinction between evaluation in the sense that we have used it above (i.e. of stance-taking and expressing an attitude) and the narrative element with the same name described by Labov and Waletzky (1967) as part of their sociolinguistic model of narrative (described below).

Although the potential for narratives to perform evaluative functions in discourse is widely acknowledged, it is also relatively under-explored compared to other areas of linguistic research on narrative. Cortazzi and Jin (2000) conceptualise the relationship between evaluation and narrative as existing at three distinct but related levels: (i.) the presence of evaluation in narrative, (ii.) evaluation of narrative (e.g. by teller and audience), and (iii.) evaluation through narrative, which orients to the ways in which tellers use narratives as a way of evaluating a person, situation or thing, or indeed the ways in which audiences can evaluate tellers on the basis of the narratives they relay. The analysis reported in this article orients to the third level; that is, it explores the ways in which patients perform one social practice - evaluation - through the use of another - narrative. Interpreting evaluation in the functional sense can be more challenging than simply its structural sense, as the former does not have any specific set of forms associated with it and can occur at any point throughout the narrative structure.

While patient feedback has been widely researched, the majority of studies have been concerned with either assessing the reliability of the feedback tools or the feedback itself, or exploring whether and how insights from feedback exercises 
are integrated into healthcare planning (see Baker et al., 2019 for a review). The only linguistic research of patient feedback carried out to-date, and thus the only research to explore not only what feedback is given but crucially how it is given in terms of language use, was carried out in previous work on the dataset examined in this article (Brookes \& Baker, 2017; Baker et al., 2019). Yet comments in this corpus have yet to be explored from a narrative perspective; we do not know whether, how and for what purposes patients might use narratives in their feedback. As an evaluative genre, patient feedback thus provides a good opportunity for exploring how narrative and evaluative social practices intersect.

\section{Data and analytical approach}

\subsection{Data}

Our corpus consists of patients' comments about the NHS posted to the NHS Choices online service (see Baker et al., 2019). NHS Choices has a number of functions, but our interest lies in the facility it offers to leave comments about service experiences. The "Friends and Family Test" (FFT) section of the site provides patients with a quick and anonymous way to give their views after they, a relative or friend have received NHS treatment. The FFT can be completed using an online form up to two years following treatment.

The FFT provides a means for giving both quantitative and qualitative feedback. Quantitative feedback is represented by a Likert scale of 1 to 5 . A rating of 1 indicates that the person would be "extremely unlikely" to recommend the service or provider to friends and family, while a rating of 5 indicates that they would be "extremely likely", with ratings of 2, 3 and 4 somewhere in-between. The rating system is followed by "Your Review", which gives patients the opportunity to provide qualitative feedback and to explain the scores they gave. This item comprises two free-text boxes. The first asks for a review "title" (max. 6o characters) and is accompanied by the prompt, "Please write a short headline summary", while the second text box asks for "The main text of your review" (max. 3,000 characters) and is accompanied by the following, more detailed guidance:

Give an account of the experience you are commenting on and give your opinion about it in your own words. The more detail you can give, the more useful your review will be. If you write a review, you will need to add a title (above) as well. Thank you for taking the time to review honestly and responsibly.

This section includes a warning that comments will not be published and may be edited if they contain identifying features (including individuals' names or gen- 
der), use abusive or "inappropriate" language, or relate to the issue of clinical negligence. These are important guidelines to bear in mind, since although our analysis of the comments suggests that some commenters break these rules, they nevertheless have the potential to influence the types of language used in the comments. After moderation, the comment is then made publicly available on the NHS Choices website, with the idea that other patients can use this information when deciding on which providers to use. Once a comment has been made public, a representative from the NHS, whose role it is to respond to feedback, may provide a reply. This can also be viewed by the public on the site.

In terms of limitations, while corpus data often provides larger and more widely representative data than the types of datasets collected in qualitative studies, the comments in our corpus can still only provide a limited picture of patient experiences as a whole. We also acknowledge that our data does not represent the experiences and perspectives of all NHS patients. Most people who use the NHS do not leave feedback, and those who do can also do so in ways other than through the NHS Choices website, for example through pen-and-paper comment boxes or by speaking to healthcare staff. What our data does represent is the feedback provided by a self-selecting sample of commenters (patients and others) who have decided to go online to provide feedback about their or another's experiences of NHS services. Because we do not possess demographic metadata for the commenters, we cannot be sure that our data provides a balanced representation of patients in terms of their identity. There may also be reasons why some individuals go online and provide feedback that are not applicable to the rest of the population. Thus, we should be mindful that the perspectives of those in our data do not necessarily represent those of all NHS patients.

Our corpus contains approximately 29 million words of comments and around 11.7 million words of responses (Baker et al., 2019). However, for the purposes of this article we focus on a smaller sample of 500 comments $(79,027$ words), comprising 100 randomly selected comments from each of the five ratings. While in reality commenters were most likely to provide positive scores, balancing our sample in terms of these ratings allowed us to explore the possible relationships between the frequency and features of narratives and the ratings being given in a context where they were afforded roughly equal weight. A breakdown of the number of words in each section of our sample, grouped by rating, is given in Table 1.

Text size generally decreases as the ratings go up, i.e. the more positively the commenter evaluated the services numerically, the briefer their comments were likely to be, with comments giving the lowest score (1) being almost twice the length, on average, of comments giving the highest score (5). 
Table 1. Word counts of texts in sample, grouped by rating

\begin{tabular}{lccc}
\hline Rating & Texts & Words & Average words per text \\
\hline 1 & 100 & 19,086 & 190.86 \\
2 & 100 & 17,249 & 172.49 \\
3 & 100 & 17,715 & 177.15 \\
4 & 100 & 14,857 & 148.57 \\
5 & 100 & 10,120 & 101.20 \\
Total & 500 & 79,027 & 158.05 \\
\hline
\end{tabular}

\subsection{Analytical approach}

We began by manually identifying all texts in our sample that contained a narrative using Labov and Waletzky's (1967) criteria that a narrative should contain at least two narrative clauses (i.e. clauses containing a verb in the simple past tense or historic past tense) and the compulsory structural element of Complication (described below).

Qualifying texts were then read and manually annotated for the presence of the narrative components of Labov and Waletzky (1967): Abstract; Orientation; Complication; Evaluation; Resolution; Coda. To analyse our texts, we manually encoded the narratives, using XML markup, to demonstrate where these elements occurred in the feedback we were studying. These searchable annotations (which we will call tags) mark the beginning and end of specific components (e.g. Abstract begins with $<\mathrm{ABS}>$ and ends with $</ \mathrm{ABS}>$ ), as well as the beginnings and ends of narratives more widely (i.e. $\langle$ NAR $>$ to $</ N A R>$ ). Below is an example of a fully annotated text.

$<$ text id=“265248” > <nar > <abs > Very rude Doctor - poor bedside manner, abrupt and sharp $</$ abs $>$

$<$ ori > I currently suffer with multiple myeloma,and was up all last night with pain which I did not know if it was related to my current condition or if it was something entirely different. In order to find out if this was treatable quicklt to relieve my pain and anguish, I visited the walk in centre in [anonymous] at 10.ooam approx today. The staff were very helpful and I was seen after waiting approx an hour as there was no doctor in at the time. </ori $>$

$<$ com $>$ When I saw the doctor, and tried to explain the pain, he was rude and abrupt, did nt give any sense of care and made me feel uncomfortable and upset, like I was wasting 
his time and it made me feel like nobody cared. He said 'ive only got 10 minutes to sort this out' and seemed more interested in turning me away then anything else. $</$ com $>$

$<$ eva $>\mathrm{He}$ was very unpleasant and a poor representation of the NHS. </eva >

$<$ res $>$ Because of his attitude, I didnt feel like answering any of his questions and just wanted to $c r y .</$ res $>$

$<\operatorname{cod}>$ I felt I had to write this as this doctor needs some feedback as he has many areas of interpersonal skill he can definitely work on. I wont be using the centre again because of this and I would appreciate some feedback to let me know this has been addressed. $</$ cod $></$ nar $></$ text $>$

The annotation of the texts was initially undertaken by two of the authors. The annotations were then checked for consistency by the others authors. Adding these annotations allows us to (i.) easily quantify the structural elements; (ii.) identify patterns they form and (iii.) exploit searchable entry points for close, qualitative analysis of narrative practices in context. The tags provide a "map" of the narratives in our data and pinpoint areas of interest for subsequent qualitative analysis. Hence we used these searchable tags as a means of studying the narrative elements quantitatively in terms of their presence and sequential patterning and qualitatively in terms of the social practices, including evaluative ones, that specific elements and sequences of elements performed, in context. This combination of quantitative insights and close, qualitative analysis is the guiding principle for the corpus-based approach taken here. The tags were searched and analysed using AntConc (Anthony 2020).

The analysis is organised according to the six narrative elements introduced above: Abstract, Orientation, Evaluation, Complication, Resolution and Complication. Crucially, our analysis orients to the ways in which narrative elements relate to the elements that tend to precede and follow them across the narratives. To facilitate this structural focus, we order the elements to reflect the sequence in which they typically occur within the narratives. However, we begin our exploration of these texts with a quantitative overview of the frequency of narratives and specific narrative elements.

\section{Analysis}

Of the 500 texts in our corpus, $302(60.4 \%)$ contained narratives and the remaining 198 (39.6\%) did not. The texts making up this $39.6 \%$ could contain elements of narratives but did not meet the criteria to be judged as fully formed narratives (see 
3.2 above). This provides an initial indication of how representative narratives are in the context of online patient feedback in general. Narratives are a fairly characteristic feature of this genre of organizational communication, being more likely to occur than not, implying that narrative is likely to play an important function in the social practice of online healthcare evaluations, or at least that this way of constructing feedback has become somewhat conventionalised.

When we divide the data up according to evaluative ratings, we find that the presence of narratives seems to correlate with negative feedback (see Table 2).

Table 2. Narrative texts per rating

\begin{tabular}{lcc}
\hline Rating & Texts with narratives (out of 100) & \% of total narrative texts \\
\hline 1 & 76 & 25.17 \\
2 & 64 & 21.19 \\
3 & 67 & 22.19 \\
4 & 56 & 18.54 \\
5 & 39 & 12.91 \\
\hline
\end{tabular}

The lowest rating (1) exhibited narratives in approximately three-quarters of the comments and accounted for a quarter of the narratives overall. Comments rated 2, which are still negative, and those rated 3, which might be considered average, are comparable in terms of their propensity to contain narratives. However, when we consider positive feedback, the number of comments containing narratives dips to 56 for feedback rated 4 and to 39 for feedback rated 5. When we compare the comments at either end of the scale, we can see that the most negative feedback contained almost twice as many texts with narratives as the most positive feedback.

We now address each structural element in turn, exploring the evaluative (and other) functions that each performs in the context of online patient feedback.

\subsection{Abstract}

Abstracts were present in $98.21 \%$ of the narratives overall. There were no large differences between the different ratings, with abstracts being present in the vast majority of narratives across all ratings: 1 (97.93\%), 2 (95.31\%), 3 (100\%), 4 (98.21\%), 5 (100\%). Therefore, the presence of Abstract does not seem to be influenced by the type of feedback given. Across all ratings, Abstract always occurs as the initial element within the narratives. This prevalence of Abstract, and its placement in text initial position, shows that the commenters are aware of the 
tellability of their narrative, a notion inherent in Labov and Waletsky's approach which recognizes "that narrative structures have two components: 'what happened and why it is worth telling"' (Bruner, 1991:12). In the Abstract of the narrative, commenters note 'why it is worth telling' by highlighting "culturally salient material generally agreed upon by members of the producer's culture to be selfevidently important" (Polanyi, 1979:207) as shown clearly in examples such as 2 and 4 below.

Qualitatively analysing the Abstracts, we found that in all ratings these elements performed similar functions. This included signalling the particular service or provider that the comment (and, so, the narrative) is about (see Example (1) below). Note, the numbers in square brackets accompanying extracts denote the rating accompanying the comments from which they are taken.

(1) Podiatry Customer Service?

A negative evaluative function is hinted at and even performed subtly by the question mark in the Abstract. Some Abstracts performed a more explicitly evaluative function, for example one commenter provides the Abstract "Bad service." As well as highlighting which provider or aspect of services that are being reviewed in the feedback, the Abstract can also function to provide an evaluation of those providers or services.

(2) Huge Waiting List and Unhelpful Staff

(3) Review of [anonymous] eye centre

(4) Excellent care

The Abstracts can also denote a particular aspect of service provision which is then the subject of the narrative and the feedback. For example, in narratives within comments rated $1-3$ we find abstracts which indicate that the narrative is about to provide a complaint about waiting times.

An area of provision that is particularly characteristic of the focus of the narratives in the feedback regards mental health services. The phrase "mental health" occurs 9 times, all in Abstracts in narratives from comments providing the lowest rating of 1 and are used mostly ( 7 occurrences) to mark out mental health services or a mental health team for negative feedback. Finally, the Abstracts (regardless of rating) can also perform a meta-discursive function by labelling the narratives in terms of their evaluative functions (see Example (3) above).

Abstracts in online patient feedback are therefore used to indicate the particular provider or aspect of the service that the narrative is about and to summarise the feedback. The Abstract sections of the narratives can also perform an evaluative function in and of themselves through explicit use of evaluative adjectives like 
"poor" and "excellent". These functions were observable in Abstracts in all ratings, though waiting times and mental health services seem to constitute a particular area of focus for the narratives in low ratings, which is consistent with wider findings that this area of provision tends to be evaluated overwhelmingly negatively by patients (Baker et al., 2019). As the examples provided here attest, the Abstracts tend to be very short in length. Indeed, they are the shortest of all the narrative elements across our data, being just 5.48 words on average. This is not particularly surprising, given that their function is to succinctly summarize aspects of the main narrative. The format of the medium is influential here; as noted earlier, the FFT provides a separate, character-limited textbox in which patients can provide a "title" for their feedback. This element seems to account for the vast majority of our narrative Abstracts. This demonstrates how both narrative and evaluative social practices can be shaped at the contextual level by the medium through which they are accomplished; in our case, the patients seemed to interpret the "title" section of the feedback form as the space into which to place an Abstract for their narratives.

\subsection{Orientation}

The next element we consider is Orientation. Orientations were not as pervasive as Abstracts but nevertheless occurred in $92.88 \%$ of narratives. Comparing the presence of Orientations across different levels of evaluation, we found that Orientations were slightly less prevalent in the more extreme forms of evaluation, occurring in $89.47 \%$ of narratives in comments rated 1 and $87.18 \%$ of narratives in comments rated 5. On the other hand, for feedback, rated 2, 3 and 4, Orientations were present in $96.88 \%, 98.51 \%$ and $92.86 \%$ of narratives, respectively.

Orientation directly follows Abstract more than any other narrative element across all feedback levels. This is the case for $83 \%$ of instances of Orientation overall, with the lowest case being in comments rated 1 (71.26\%) and the highest being in comments rated 3 (97.01\%) but the level of evaluation does not seem to influence whether and the extent to which Orientations follow Abstracts, as it followed Abstract more than anything else at all feedback levels.

Through Orientations, patients describe the circumstances (time, place) and people involved in the episode of care at the heart of the narrative. For all ratings, this can also involve descriptions of the health problems that led the patients to require care in the first place. In the case of comments rated 1, the Orientations can contribute to patients' identity construction, as patients use this element to present themselves as long-term patients of a particular practice before then going on to describe a negatively evaluated episode of care, as in this example where we 
have included the Orientation and the following Complication. We have left in the annotation to show where the elements start and end.

(5) <ori> I usually find this walk in centre very useful but today I arrived at 7pm and $<$ /ori $><$ com $>$ was told that the centre was closed as it shuts early on the first Thursday of every month. However, I can not find any reference to this online on any website and the receptionist was extremely rude in explaining this. $</$ com $>$

In such cases, Orientations can help to set up a sense of expectation based on previously positive experiences, before the complication indicates ways in which this expectation has not been met in the particular episode in question, prompting the negative feedback. Simultaneously, claims of having attended a provider over a long period of time might also be viewed as contributing to the construction of an expert patient identity to further legitimate the evaluation given.

Not all Orientations described long-term experiences with a provider, though, and in comments rated 4 and 5 in particular, some patients described having to attend a provider at short notice due to a medical emergency or their usual practice not being available. Such cases tend to occur in the positive ratings because patients then positively evaluate the care they received at short notice:

(6) I used the services of [anonymous] walk-in centre on the recommendation of a friend, as I was unable to get an appointment with my GP for 5 days.

In this sense, then, Orientations can be used to set up expectations which contribute to the social practice of evaluation. In the negative comments, the Orientations can set up high expectations (based on long-term positive experiences) that are then not met in the particular episode of care being evaluated. Meanwhile, in the positive comments, patients can use Orientations to describe requiring care at short notice, which sets up low expectations that are then exceeded, thus triggering positive evaluation.

However, Orientations were also slightly less pervasive in the very positive and very negative comments, which might suggest that this element becomes more optional in these cases, with some patients sacrificing background detail to get to the heart of the praise or complaint, which is realised through the Complication and Evaluation elements.

(7) <abs $>$ Furious $<$ /abs $><$ com $>$ You sent me home my waters had broke. I was in agony I could not stand sit or lie down. I threw up several times and you told me I wasn't in labour without assessing me because I couldn't feel my baby. </com> 
(8) <eva> I was so impressed with the service here. Receptionists were polite and friendly, $</$ eva $><$ com $>$ my daughter was seen very quickly by one of the best nurses I have encountered $</$ com $>$

Although Orientations were slightly less frequent in these more extreme forms of feedback, we should bear in mind that they were still present in the vast majority of narratives at all levels of feedback, including those rated 1 and 5 .

\subsection{Complication}

As a compulsory element, Complication was present in all the narratives in our data, where it tended to follow Orientation more than any other element (average: $48.4 \%$ of cases), though it also often follows Evaluation (average: $42.52 \%$ ). As the Table below shows, Complication follows Orientation more than any other element in feedback rated between 1 and 4 but for comments rated 5, the Complication follows Evaluation the most. In all cases, though, Complication follows Orientation and Evaluation either most or second-most often and, taken together, these account for the vast majority of uses of Complication for all ratings.

Table 3. Elements which precede Complication across the comments (expressed as a percentage of cases of Complication)

\begin{tabular}{lll}
\hline Level & Most often & Second most often \\
\hline 1 & Ori-Com $(49 \%)$ & Eva-Com $(25.17 \%)$ \\
2 & Ori-Com $(48.02 \%)$ & Eva-Com $(47.37 \%)$ \\
3 & Ori-Com $(51.82 \%)$ & Eva-Com $(44.55 \%)$ \\
4 & Ori-Com (53.16\%) & Eva-Com $(40.51 \%)$ \\
5 & Eva-Com (55\%) & Ori-Com $(40 \%)$ \\
\hline
\end{tabular}

Complication, therefore, has a strong association with the Orientation and Evaluation elements and, as Section 4.4 will show, it precedes Evaluation more than any other element across all levels of feedback.

In particular in comments providing rated 1 and 2, Complications concern events during the episode of care that have driven the patients' negative appraisals. As indicated by the Abstracts examined earlier, these events typically concern waiting lists but also cases where patients are given advice by practitioners which proves to be inaccurate or inconsistent. Such Complications are conveyed through reporting verbs in perfect past-tense, such as told, requested, asked and refused. 
(9) I was told by the nurse "we don't do anything for eye ulcers or cysts". The nurse didn't bother to check my eye to see what was wrong with it

By contrast, the narratives in positive comments rated 4 and 5 featured Complications which alluded to the health problems that patients experienced, which were then judged to have been adequately dealt with through care.

(10) I was having problems breastfeeding my daughter, after she had developed breast aversion following the techniques used at [anonymous]. The staff at [anonymous] were supportive and patient in trying to reverse this, sharing their personal and professional experiences to help me find what worked for us

The Complications in feedback rated 3 are more like those in the negative comments than the positive comments, as they describe experiences that are negatively appraised. The difference between the comments giving low and middling scores is that the negative experience is balanced out by positive experiences with the same provider in the past. For example, the extract below is a Complication from a comment in which the patient concludes that: "this was my third visit and by far the worst".

(11) not one person could actually tell me what procedure had been done on my knee. the surgeon never once came to see me after the operation. after moving me to a ward after the op into a single room no one checked on me for over three hours, they may have looked through the door but not one observation was noted in my notes.

As noted, Complications seem to be strongly associated with Evaluations as well as Orientations. We now move on to consider the functions of Evaluations in the narratives, paying particular attention to how they relate to Complications.

\subsection{Evaluation}

Evaluations were present in approximately three quarters of all narratives (74.50\%). When we consider the presence of Evaluations across the different levels of feedback, we find that this element is more common in comments giving positive feedback. Specifically, Evaluations were present in $87.18 \%$ of narratives in comments rated 5 and $76.79 \%$ of narratives rated 4 but $70.31 \%$ of narratives rated 2 and $71.05 \%$ of narratives rated 1 . Evaluations were least frequent, relatively speaking, in narratives in feedback rated 3. So, Evaluations seem to be more characteristic of feedback that is either positive or negative compared to more moderate feedback, being particularly prevalent in very positive feedback. 
Across all feedback levels, Evaluation followed Complications more than any other element ( $49.53 \%$ of cases on average). This pattern was stronger for narratives in negative comments (rated 1 and 2; average 51.81\%) and middling comments (rated $3 ; 62.92 \%$ ) than more positive feedback (rated 4 and 5 , average $40.57 \%)$.

Regardless of the element that it follows, the Evaluations at all feedback levels can be characterised as providing an appraisal of the services and experiences being described. While this evaluative function could, as we have seen, be performed potentially by any of the narrative elements, the social practice of evaluation was most explicitly fulfilled by the narratives' structural Evaluations.

(12) I was terrified.

(13) The staff were friendly and put me at ease during the appointment. Unfortunately the waiting involved during an already stressful time in my opinion is unacceptable.

(14) I was very impressed! The clinic is clean and tidy, the reception staff were very polite, curteous and pleasant to talk to

The Evaluation elements are particularly revealing in narratives within comments rated 3 , as these comprise a mixture of positive and negative appraisals, which helps to explain the score given, as patients describe a mixture of positive and negative experiences.

The Evaluation component, as conceived by Labov \& Waletzky (1967), is not necessarily evaluative in the sense that it does not necessitate patients appraising or taking a stance on the events being described. The tendency for the Evaluations to fulfil evaluative social practices in our patient feedback narratives should therefore not be interpreted as a given. Rather, it reflects the fact that the "point" of the texts in this genre - their raison d'être - is to appraise healthcare experiences.

\subsection{Resolution}

Resolutions tended overwhelmingly to follow Complications. This was the case for $74.17 \%$ of instances of Resolutions across all feedback levels. Resolutions occurred in $77.66 \%$ of the narratives, where they exhibited a slight preference for comments accompanying lower feedback ratings. Indeed, Resolutions were most frequent in narratives within comments accompanying ratings of 1 (82.89\%), followed by those rated $2(78.13 \%)$. The differences in the percentages are not too large, though, as Resolutions were present in $76.12 \%$ of narratives in comments rated $3,76.79 \%$ of those rated 4 and $74.36 \%$ of those rated 5 . The largest difference 
here is between comments at either end of the pole, then, with Resolutions occurring in $8.53 \%$ more of the narratives in the comments rated 5 compared to 1.

In feedback rated 1 and 2 , the resolutions to the narratives tend to be that the patient's health issue remains unresolved, that they are still requiring or waiting for care or that the patient has managed to receive the service they needed from a different provider (see Example (15)).

(15) Luckily the medical centre next door was more sympathetic to my post birth incontinence issues and let me use their toilet.

In comments providing scores of 3 , Resolutions do not describe health problems being unresolved. However, patients frequently remark on how long they have had to wait to receive treatment and thus to reach a positive health outcome, which could help to explain why these comments gave middling as opposed to positive scores, as the health outcomes are ultimately positive but have involved a wait that is deemed by the patient to be overly long.

(16) However, after much asking and waiting the medication arrived at $6.00 \mathrm{pm}$ after which I took her to my home to care for her, a journey which takes 1 hour.

These types of Resolutions were also evident in narratives within comments rated 4 , in addition to those which straightforwardly describe their health problems as having been resolved by a particular provider. This latter type of Resolution was characteristic of those found in the narratives from comments rated 5.

\subsection{Coda}

Codas were observed in $77.71 \%$ of the narratives, so are one of the less frequent elements, aligning with the view of Labov and Waletzky (1967) that Codas are one of the more optional elements in canonical narratives. Codas are most frequent in positive feedback, occurring in $87.18 \%$ of the narratives in comments rated 5 and $82.14 \%$ in those rated 4 . Codas are slightly less frequent in the worst feedback, occurring in $78.95 \%$ of narratives in comments rated, $65.63 \%$ of those rated 2 and $74.63 \%$ of narratives in comments rated 3 . So, Codas are particularly characteristic of very positive feedback, being observed in $8.23 \%$ more of the narratives in comments rated 5 compared to those rated 1 , which could explain their relatively low frequency overall, as narratives are, as we have seen, more characteristic of negative feedback.

Codas tend to occur at the very end of the narratives and follow Resolutions more than any other element (average: $50.42 \%$ ). A feature of the Codas at all levels of feedback is that patients frame the past events that have been recounted 
in more generalisable terms as being applicable to the present and to others beyond themselves and their own experiences. In the negative feedback, problems described in the Complication are framed as being symptomatic of broader issues within the NHS.

(17) The NHS is ailing and needs treating- not good enough!

These elements are also characterised by messages directed to other patients who are imagined readers in these scenarios. This manifests in a pronounced frequency of second-person pronouns, particularly you and your, relative to other levels of feedback. And these can be used in general reference:

(18) Go in expecting to be called a liar, denied treatment, called names and not get taken seriously and you sure won't be disappointed. Change in management here is definitely needed!

Codas are also characterised by the use of speech acts (Austin, 1962). In comments rated 4 and 5, patients use Codas to recommend a provider to other patients and, in Codas directed at the providers themselves, to express thanks for the positively appraised standards of service.

(19) the whole experience was pleasant and extrmely efficient, I would certainly recommend this centre and should I need help again in the future this will be my first choice! Thank you for an impressive experience.

On the other hand, speech acts in Codas in comments rated 1 and 2 tend to be directed just to other patients rather than providers and recommend that patients avoid a particular provider on the basis of their own negatively evaluated experiences.

(20) I would not recommend this place to my worst enemy. Go to your GP or A\&E instead.

As well as orienting to their own future intentions, then, the Codas in the feedback can indicate the "imagined" audience of the comments (Bell, 1984). Here we have noted a distinction between the positive and negative comments. While positive comments could be addressed to the provider and other patients or users of the website, the negative comments tended to be addressed just to the latter, with the providers talked about rather than to. In terms of social practices, then, the Codas in negative comments can contribute to the social practice of evaluation through linguistic choices which render the experiences described and evaluations provided as being generalisable beyond the experience of that patient alone, thus arguably framing them as more pressing in nature, reflecting wider problems with the healthcare system. In positive comments, on the other hand, Codas con- 
tribute to the social practice of evaluation through the use of speech acts such as thanking and recommending. Yet the use of direct address in Codas, directed both to providers and to other patients, also contributes to the construction, in the former case, of a positive relationship between the patient and the provider and, in the latter case, of an "imagined community" of patients with common values who will likely share each other's views on what counts as good and bad care.

\section{Discussion}

This paper has provided a range of insights into the intersection of narrative and evaluative social practices in the context of online patient feedback on healthcare services. In response to question (i.) How prevalent is narrative in online patient feedback?, our analysis has shown that the social practice of narrative is fairly pervasive in this type of text. Although none of the patients are explicitly told to provide narratives on the NHS Choices website, they nevertheless provide evaluation through the use of narratives in approximately $60 \%$ of cases. This could indicate a relationship between the social practices of narrative and evaluation in this context, perhaps even to the extent that the use of the former to accomplish the latter has become a conventionalised way of providing feedback in this context.

Yet our analysis has also shown that patients are more likely to construct their experiences of healthcare services using narrative when evaluating those experiences negatively. One explanation for this could be that the narrative social practices in which these patients engage allow them to provide evidence to support their claims about poor service provision, which are more likely to be challenged than claims about good service provision. For example, the narrative social practices observed in the patients' comments could also contribute towards the construction of an experienced patient identity to legitimate negative feedback, as well as being used to construct an imaged community of patients who will share values and priorities, perhaps for the purpose of framing negative evaluations as being generalisable beyond the immediate experience of the patients themselves. Another possible explanation relates to our previous findings relating to this data; specifically, Baker et al. (2019) reported that while the most positive feedback tends to be given for consistently high standards of service over a long period of time, the lowest ratings could be given for just a single negative experience. With this in mind, it could be the case that descriptions of single negative experiences lend themselves more naturally to a narrative style, as conceptualised for the purposes of this study, than descriptions of multiple positive experiences over a long time. 
In answer to question (ii.) Which structural narrative elements are used to perform evaluative social practices?, we have found that evaluative practices within healthcare feedback can be fulfilled by any or all structural elements of narratives, with different parts of the narrative also being able to function in combination to evaluate healthcare services. Abstracts indicate the particular provider or aspect of a service that the narrative is about but can summarise the point of the feedback, sometimes providing explicit evaluations at the same time through positively or negatively valenced adjectives. Orientations can contribute to evaluative practices by setting up expectations which, in the Complication, are not met in the negative feedback, but which are exceeded in the positive feedback. Orientations could also contribute quite explicitly to the construction of positive patient identities, for example as long-standing, expert patients who are experienced and qualified enough to provide a legitimate and reliable evaluation of the provider in question. In positive feedback, Complications tend to refer to health issues that are then resolved, while in negative feedback they denote some aspect of provision which triggers a negative evaluation, such as a long wait or being given bad information. The Evaluation elements tend to sum up the point of the narratives. Through Evaluations, patients often expressed their stance towards the provider or the events described, and so in many ways provided the most explicit indication of their appraisal of the services. As noted, while Evaluations, as described by Labov and Waletzky (1967), do not necessarily constitute evaluative social practices, the fact that they could be characterised as such in our data is likely a reflection of the evaluative purpose of the patient feedback genre. In the Resolutions, we observed differences at the various levels of feedback. In negative feedback, Resolutions tend to describe unresolved health issues and continued waits for care, while in both middling and positive feedback they describe the ways in which the health problems noted in the preceding Complications have been resolved. Finally, occurring at the end of narratives, Codas resulted in speech acts such as thanks and recommendations in positive feedback and warnings to others to avoid a particular practice in negative feedback. These speech acts have the potential to contribute not only to the social practice of evaluation but also to the construction of relationships between patients and their providers, as well as the construction of an "imagined" community of patients with shared values.

In terms of research question (iii.) Do narrative structures alter depending on the evaluations they perform (i.e. as positive or negative)?, we have found that while patients did not necessarily have to conform to the canonical narrative structure described by Labov and Waletzky (1967), most of the narratives did indeed resemble this structure. We found that the structure of the narratives did not seem to change depending on whether the evaluative social practices were used to provide positive or negative appraisals, with the only exception being that 
Complications were slightly more likely to follow Evaluations in the most positive feedback, whereas they were most likely to follow Orientations at all other levels of feedback. However, this is a relatively minor variation and, overall, the narratives were largely uniform in structure, regardless of rating.

In line with the view of narrative and evaluation as forms of social practice set out at the beginning of this paper, the narratives and evaluations explored in the previous section are, crucially, situated within particular textual, but also socio-cultural, contexts which constitute them. Regarding their textual context, the affordances of the NHS Choices website means, for example, that comments are text-only, while the affordance of a title section preceding the free-text comment box meant that patients tended to place the Abstracts of their narratives there. We have also found evidence that the public nature of the medium affords a particular form of social practice, as patients directed their comments not only to the providers in question but also to other members of the public visiting the NHS Choices website. The latter necessitated the construction of an "imagined" community of patients with shared values and needs.

The Codas were, as noted, particularly telling regarding the commenters' intended or "imagined" audiences, and here we noted a distinction between the audiences constructed for positive and negative comments. While positive comments were directed at both providers and other patients or website users, the negative feedback was seemingly intended for other patients rather than being explicitly directed at the providers themselves. Yet we should also be mindful of the possibility that patients craft such negative comments to ensure that they are read by the providers. But excluding them from the Codas and speaking directly to other patients constitutes the performance of a kind of consumer empowerment, with the patients cutting providers out of the process and expressing their dismay by taking their "business" elsewhere and encouraging others to do the same. This can be interpreted as keying into a customer service discourse that is characteristic not only of the language of online consumer reviews but also of the UK healthcare landscape in general (Brookes \& Harvey, 2016a) which is becoming more characteristic of the NHS in England. Indeed the practice of patients giving feedback has itself attracted criticism for the perception that it can create a set of expectations which put patients in a customer role, potentially contributing towards a marketization of discourse regarding the NHS (ibid.; Baker et al., 2019). This is evident in the title of the website itself, NHS Choices, in which the word "choices" denotes a system of options from which patients can choose, with the implication being that providers and practices can be distinguished in terms of the quality of service they provide. Quality of service is indicated by the ratings and reviews that patients provide, with the intention that this will create competition between healthcare providers, as patients choose the providers with the high- 
est ratings. Such contextual factors could be significant, particularly if it is indeed the case that such macro level socio-political changes to the UK healthcare landscape, such as the increasing privatisation of UK healthcare services (Davis et al., 2015), are refracted through - indeed even constituted by - the kinds of narrative and evaluative social practices in which patients engage when posting their public comments on the NHS Choices website.

\section{Conclusion}

This article has contributed new insight into the frequency and structure of narratives in online patient feedback and how narrative practices in this context contribute to the social practice of evaluation. Narratives are fairly pervasive in the context of online patient feedback on healthcare services, but they are also more likely to occur in negative, rather than positive, feedback. However, their structure does not seem to be influenced by whether the evaluation accomplished by means of it is positive or negative, and all structural elements of narratives could contribute to evaluative social practice, in their own right and/or in combination with other elements. We have argued that these trends may reflect a conventionalisation of narrative practices in the activity of feedback provision, with the tendency for negative feedback to focus on single episodes of care (as opposed to longerterm experiences) being particularly amenable to narrative style. For practitioners and feedback monitors, it is important to bear in mind that while narrative practices can contribute to both positive and negative evaluative social practices, they are, based on our data, much more likely to contribute to the latter. On a practical level, this could mean that evaluations elicited as narratives (for example, with invitations to patients to tell "stories") may be more likely to engender negative as opposed to positive evaluations.

We have also found evidence that the narrative social practices that constitute the patient feedback examined here could perform other social functions which may contribute to their evaluative force. For example, through the narrative practices they engage in when providing feedback, patients could construct themselves as longstanding and experienced (even expert) patients who are thus suitably qualified to evaluate healthcare services and whose judgments on healthcare quality are legitimate. Other social functions performed by the narratives could also contribute to evaluative social practices, for example in comments in which patients construct an imagined community of patients with shared values whose choices of healthcare provider would likely be driven by the same priorities. In positive feedback, narrative practices could also construct relationships between patients and their providers, with the former thanking the latter and rec- 
ommending them to other patients. On the other hand, narratives which performed negative evaluations were more likely to be addressed to other patients visiting the NHS Choices website, with the provider seemingly excluded from the dialogue.

We have thus argued that the narrative practices which constitute patient feedback, and which contribute to the evaluations given, are shaped by the particular textual and socio-cultural contexts in which they are situated. At the textual level, the public nature of the NHS Choices platform may engender the aforementioned types of narrative practices which construct an imagined community of patients, with the comments accordingly being directed at them and seemingly being written for their benefit. Indeed, in our previous analysis of this data, we have similarly found evidence of patients writing comments seemingly for other patients, for example using humour at the expense of the provider in question (Baker et al., 2019). At the same time, the social practices, both narrative and evaluative, observed in this analysis can be viewed as both being shaped by and shaping their socio-cultural contexts in terms of the UK healthcare landscape in which they are situated. Narratives designed to recommend providers to patients, or likewise to warn other patients off a particular provider, can be interpreted as enacting the role of informed healthcare consumer, as well as attributing that role to other patients visiting the site. While reflecting the dynamics of an increasingly privatised UK healthcare landscape, the public nature of these comments, being read by other patients and potentially informing their own healthcare decisions, can also contribute to this ongoing process of commercialisation. We would argue that it is therefore important for feedback monitors to be mindful of the potential for narrative practices constituting feedback to be shaped not only by the particular medium in which the feedback is given but also by wider socio-cultural trends which, at present, may contribute to a more consumerist approach to healthcare evaluation on the part of patients.

On a methodological note, existing approaches to narrative in illness and health(care) contexts have, as noted, attracted criticism for having to rely on relatively limited datasets that restrict the generalisability of the findings, with the narratives gathered not necessarily being representative of the wider population or context under study (Ziebland, 2013). We feel that the method we have used in this paper represents a step in the right direction for linguistic research on healthcare narratives, as it utilises annotation techniques in conjunction with Labov and Waletzky's (1967) established narrative framework, to enable analysis of narrative patterns and functions in larger and more generalisable datasets than would be practical using a purely manual approach. In health(care) organizational contexts, the use of annotation and larger datasets could help researchers and practitioners alike to bridge the gaps between social and medical sciences generally (Brookes 
\& Harvey, 2016b), and narrative and evidence-based medicine specifically. The approach we have taken in this paper could, in theory, be applied to any type of textual data where narratives are present. Here we have focused on narratives of healthcare experience, but analysis utilising the annotation of narrative elements could also be used to compare and explore the functions of narrative elements in texts related to other organizational contexts. Indeed, feedback mechanisms like FFT from which our data derives are redolent of other user/consumer feedback tools that are used in a seemingly increasing range of organisation contexts that are both public service and commercial in nature. The ways in which individuals provide evaluations and perform their identities as consumers is thus likely to garner increasing attention from researchers interested in organisational communication in the future, as well as other areas of Applied Linguistics and Discourse Studies. For such endeavours, our analysis has shown there to be merit in focusing on the evaluative potential of narratives in such contexts, as well as demonstrating the benefits of utilising corpus linguistics approaches to do so.

\section{Funding}

This research was funded by the Economic and Social Research Council (Centre for Corpus Approaches to Social Science), grant number ES/Roo8906/1.

\section{References}

Anthony, L. (2020). AntConc (Version 3.5.9) [Computer Software]. Tokyo, Japan: Waseda University. Available from https://www.laurenceanthony.net/software

Arribas-Ayllon, M. (2021). Narrative Analysis: DNA testing and collaborative knowledgebuilding in a CFS/ME forum. In G. Brookes \& D. Hunt (Eds.), Analysing Health Communication: Discourse Approaches. Palgrave Macmillan. https://doi.org/10.1007/978-3-030-68184-5_4

Atkinson, P., \& Delamont, S. (2006). In the Roiling Smoke: Qualitative Inquiry and Contested Fields. International Journal of Qualitative Studies in Education, 19(6), 747-755. https://doi.org/10.1080/09518390600975974

Austin, J. (1962). How to do things with words. Oxford: Oxford University Press.

Bell, A. (1984). Language Style as Audience Design. In N. Coupland and A. Jaworski (Eds.), Sociolinguistics: A Reader and Coursebook (pp. 240-250). Palgrave Macmillan.

Baker, P., Brookes, G., \& Evans, C. (2019). The Language of Patient Feedback: A Corpus

Linguistic Study of Online Health Communication. Routledge. https://doi.org/10.4324/9780429259265

Brookes, G., \& Baker, P. (2017). What does patient feedback reveal about the NHS? A mixed methods study of comments posted to the NHS Choices online service. BMJ Open, 7: e013821. https://doi.org/10.1136/bmjopen-2016-013821 
Brookes, G., \& Harvey, K. (2016a). Opening up the NHS to market: using multimodal critical discourse analysis to examine the ongoing corporatisation of health care communication. Journal of Language and Politics, 15(3), 288-302. https://doi.org/10.1075/jlp.15.3.04bro

Brookes, G., \& Harvey, K. (2016b). Examining the discourse of mental illness in a corpus of online advice-seeking messages. In L. Pickering, E. Friginal and S. Staples (Eds.), Talking at Work: Corpus-based Explorations of Workplace Discourse (pp. 209-234). Palgrave Macmillan. https://doi.org/10.1057/978-1-137-49616-4_9

Brookes, G., \& McEnery, T. (2020). Corpus Linguistics. In S. Adolphs and D. Knight (Eds.), The Routledge Handbook of English Language and Digital Humanities (pp. 378-404). Routledge. https://doi.org/10.4324/9781003031758-20

Bruner, J. (1991). The narrative construction of reality. Critical Inquiry, 18, 1-21. https://doi.org/10.1086/448619

Charon, R. (2006). The self-telling body. Narrative Inquiry, 16, 191-200. https://doi.org/10.1075/ni.16.1.24cha

Clark, J., \& Mishler, E. (1992). Attending to patients' stories: Reframing the clinical task. Sociology of Health and Illness, 14, 344-370. https://doi.org/10.1111/1467-9566.ep11357498

Cortazzi, M., \& Jin, L. (2000). Evaluating Evaluation in Narrative. In S. Hunston and G. Thompson (Eds.), Evaluation in Text: Authorial stance and the construction of discourse (pp.102-120). Oxford University Press.

Davis, J., Lister, J., \& Wringley, D. (2015). NHS for Sale. Merlin Press.

Frank, A. (1995). The Wounded Storyteller. University of Chicago Press. https://doi.org/10.7208/chicago/9780226260037.001.0001

Georgakopoulou, A. (2007). Small Stories, Interaction and Identities. John Benjamins. https://doi.org/10.1075/sin.8

Goffman, E. (1955). On Face-Work: An Analysis of Ritual Elements in Social Interaction. Psychiatry: Interpersonal and Biological Processes, 18(3), 213-231. https://doi.org/10.1080/00332747.1955.11023008

Greenhalgh, T. (1999). Narrative based medicine in an evidence based world. British Medical Journal, 318, 232. https://doi.org/10.1136/bmj.318.7179.323

Greenhalgh, T., \& Hurwitz, B. (1999). Why study narrative? British Medical Journal, 318(7175), 48-50. https://doi.org/10.1136/bmj.318.7175.48

Harvey, K., \& Koteyko, N. (2012). Exploring Health Communication: Language in Action. Routledge. https://doi.org/10.4324/9780203096437

Holmes, J., \& Marra, M. (2005). Narrative and the construction of professional identity in the workplace. In J. Thornborrow and J. Coates (Eds.), The Sociolinguistics of Narrative (pp. 913-213). John Benjamins. https://doi.org/10.1075/sin.6.10hol

Jones, R. (2013). Health and Risk Communication: An Applied Linguistic Perspective. Routledge. https://doi.org/10.4324/9780203521410

Kleinman, A. (1988). The illness narratives: Suffering, healing, and the human condition. Basic Books.

Labov, W., \& Waletzky, J. (1967). Narrative analysis. In J. Helm (Ed.) Essays on the Verbal and Visual Arts (pp. 12-44). University of Washington Press.

Lamerichs, J., \& Te Molder, H. (2003). Computer-mediated communication: from cognitive to a discursive model. New Media \& Society, 5(4), 451-473.

https://doi.org/10.1177/146144480354001 
Linde, C. (1997). Evaluation as a linguistic structure and social practice. In B.L. Gunnarsson, P. Linell \& B. Nordberg (Eds.), The Construction of Professional Discourse (pp. 151-172). Routledge.

Polanyi, L. (1979). So what's the point? Semiotica, 25, 207-241. https://doi.org/10.1515/semi.1979.25.3-4.207

Polkinghorne, D. (1988). Narrative knowing and the human sciences. New York Press.

Riessman, C. (1990). Strategic Uses of Narrative in the Presentation of Self and Illness. Social Science and Medicine, 30, 1195-1200. https://doi.org/10.1016/0277-9536(90)90259-U

Sarangi, S., \& Clarke, A. (2002). Constructing an account by contrast in counselling for childhood genetic testing. Social Science \& Medicine, 54, 295-308. https://doi.org/10.1016/So277-9536(01)00029-6

Ziebland, S. (2013). Narrative interviewing. In S. Ziebland, A. Coulter, J. D. Calabrese \& L. Locock (Eds.), Understanding and Using Health Experiences: Improving patient care (pp. 38-47). Oxford University Press. https://doi.org/10.1093/acprof:0so/9780199665372.003.0005

\title{
Address for correspondence
}

Gavin Brookes

Department of Linguistics and English Language

Lancaster University

County South

Lancaster LA1 4 YW

UK

g.brookes@lancaster.ac.uk

https://orcid.org/oooo-00o3-0726-2559

\section{Co-author information}

\author{
Tony McEnery \\ Department of Linguistics and English \\ Language \\ Lancaster University \\ a.mcenery@lancaster.ac.uk \\ (iD) https://orcid.org/oooo-0oo2-8425-6403 \\ Mark McGlashan \\ School of English \\ City Centre Campus \\ Birmingham City University \\ mark.mcglashan@bcu.ac.uk \\ (iD) https://orcid.org/oooo-ooo1-9129-0848
}

Gillian Smith

Department of Linguistics and English

Language

Lancaster University

g.smith6@lancaster.ac.uk

Mark Wilkinson

Department of Linguistics and English

Language

Lancaster University

m.wilkinson7@lancaster.ac.uk 


\section{Publication history}

Date received: 24 August 2020

Date accepted: 21 July 2021

Published online: 23 August 2021 